\title{
Dissociative photoionization of the NO molecule studied by photoelectron-photon coincidence technique
}

\author{
A. Kivimäki , J. Alvarez-Ruiz , M. Coreno , M. de Simone , A. Moise , L. Partanen , \\ R. Richter , M. Stankiewicz
}

CNR-IOM, Laboratorio TASC, 34149 Tizeste, Italy

Departamento de Ouinica Líser, Instituto de Duimica-fisica Rocasolano, Consejo Superior de Investigaciones Cientfficas, Serrano 119,28006 Madrid, Spain

CNR-IMIP, Montelibretti, 00016 Rome, italy

Sinctotrone Trieste, 34149 Trieste, italy

Department of Physical Sriences, University of Onk, P.O. Box 3000, 90014 Oulu, Finland

Instytat Fizyki im. Mariana Smoluchowskiego, Uniwersytet jagielionski, $30-059 \mathrm{Krak}$ ów, Poland

Keywords:

Nitric oxide

Photoelectron-photon coincidence

Imer valence

Dissociative photoionization

vacuum ultraviolet emission

\begin{abstract}
A B S T R A C T
Low-energy photoelectron-vacuum ultraviolet (VUV) photon coincidences have been measured using synchrotron radiation excitation in the inner-valence region of the nitric oxide molecule. The capabilities of the coincidence set-up were demonstrated by detecting the $2 \mathrm{~s}^{-1} \rightarrow 2 \mathrm{p}^{-1}$ radiative transitions in coincidence with the $2 s$ photoelectron emission in $\mathrm{Ne}$. In $\mathrm{NO}$, the observed coincidence events are attributed to dissociative photoionization with excitation, whereby photoelectron emission is followed by fragmentation of excited $\mathrm{NO}^{+}$ions into $\mathrm{O}^{*}+\mathrm{N}^{*}$ or $\mathrm{N}^{*}+\mathrm{O}^{*}$ and VUV emission from an excited neutral fragment. The highest coincidence rate occurs with the opening of ionization channels which are due to correlation satellites of the $3 \sigma$ photoionization. The decay time of VUV photon emission was also measured, implying that specific excited states of $\mathrm{N}$ atoms contribute significantly to observed VuV emission.
\end{abstract}

\section{Introduction}

When a molecule absorbs radiation of energy above the first jonization potential (IP), it can be ionized or it can end up in a highly excited neutral state. The latter is often called a superexcited state. More specifically, superexcited states are either Rydberg excited states converging to higher ionization thresholds of the molecule or multiply excited states, in particular, doubly excited states. Superexcited states can decay in a number of ways, some of which produce neutral excited fragments. Therefore fluorescence emission from neutral fragments has been often used as a probe in the search of superexcited states [1]. Conversely, features observed in the fluorescence excitation functions of fragments are typically attributed to superexcited states (e.g., [2-4]). However, excitation to superexcited states is not the only mechanism that causes fluorescence emission from fragments above the first IP of the molecule, as direct photoionization may also contribute to fluorescence intensity. For example, coincidences between fluorescence photons and $\mathrm{N}^{+}$jons have been observed in the inner-valence region of the $\mathrm{N}_{2}$ molecule [5-7]. They were ascribed to events where photoionization is followed firstly by dissociation of the resulting molecular ion, $\mathrm{N}_{2}{ }^{+} \rightarrow \mathrm{N}^{+}+\mathrm{N}^{*}$, and consecutively by fluorescence emission from the excited neutral fragment $\mathrm{N}^{*}$. This process is called Dissociative photolonization [with] Excitation (D]E). It was suggested that correlation states related to inner-valence photoionization are doorway states leading to DIE [6,7]. Correlation states exist if a state with an inner-valence hole cannot be described by a single-hole electron configuration but it contains contributions from two-hole one-electron $(2 \mathrm{~h}-1 \mathrm{e})$ or even higher order states due to configuration mixing (2h-1e states have two holes in outer valence orbitals and an electron in a virtual orbital which is not occupied in the molecular ground state). The effect is manifested in the inner-valence photoelectron spectrum by the appearance of several satellite peaks instead of just one main photoelectron line. Correlation satellites can be populated at all excitation energies above their binding energies. Therefore, if a fluorescence line is due to DIE it displays a continuous intensity distribution as a function of excitation energy. On the contrary, superexcited states yield resonant features in fluorescence excitation functions.

In the present paper, we have studied dissociation of the nitric oxide (NO) molecule in the inner-valence region by a slightly different coincidence technique as in the above-mentioned 
studies of the $\mathrm{N}_{2}$ molecule [5-7]. Namely, we have observed coincidences between photoelectrons and fluorescence photons. Photoelectron-fluorescence coincidence (PEFCO) technique has earlier been used to determine the radiative lifetimes of the excited ionic states of some molecular ions [8,9]. Bloch and Turner [8] employed a He I photoelectron spectrometer to select a specific molecular ionic state and registered fluorescence emission from that state with a photomultiplier tube (PMT). In the experiments of Biehl et al. [9], a threshold electron analyzer was used to collect threshold electrons in coincidence with fluorescence photons that were detected with a PMT, equipped with a filter to isolate the wavelength windows of interest. In those studies, fluorescence occurred directly after photoionization, i.e., the molecules did not dissociate before fluorescing. More recently, Taylor and Eland [10] have reported electron-fluorescence photon coincidences for a number of diatomic and triatomic molecules in which fluorescence emission occurred in either parent or fragment ions. Their triple coincidence setup hosted a channeltron for electron detection and a PMT for photon detection in the wavelength range 200-500 nm; it also included a time-of-flight (TOF) mass spectrometer to characterize photoions. The same authors have later developed an electron-electron-photon coincidence setup, where a magnetic-bottle electron TOF (e-TOF) spectrometer was used to observe an electron pair resulting from double photoionization and the same PMT (200-500 nm sensitivity range) collected photons emitted by the $\mathrm{N}_{2} \mathrm{O}^{2+}$ or $\mathrm{CS}_{2}{ }^{2+}$ dication $[11,12]$. In the present work, we have exploited yet another electron-photon coincidence scheme: installed at a synchrotron radiation source, a conventional e-TOF spectrometer was used to observe slow electrons in coincidence with VUV photons $(\lambda<150 \mathrm{~nm})$ that were collected with a micto-channel plate (MCP).

If we hypothesize that a NO molecule undergoes DIE (as it will be argued later), the electron and photon emissions are separated by dissociation of the molecular ion. For NO, DIE can be written schematically as follows:

$$
\begin{array}{rlrl}
\mathrm{NO}+\mathrm{h} v \rightarrow \mathrm{NO}^{+*}+\mathrm{e}^{-} \rightarrow & \mathrm{N}^{*}+\mathrm{O}^{+}+\mathrm{e}^{-} \text {or } \mathrm{O}^{*}+\mathrm{N}^{+}+\mathrm{e}^{-}, \\
& \downarrow & \downarrow \\
& \mathrm{N}+\mathrm{h} v^{\prime} & \mathrm{O}+\mathrm{h} v^{\prime \prime}
\end{array}
$$

where the asterisk $\left(^{*}\right)$ indicates an excited species. Photon absorption causes the prompt electron emission, while a fluorescence photon is emitted after the fragmentation of the molecular ion. This type of experiment is therefore affected by two time constants: that of molecular dissociation and that of the lifetime of the emitting state. The dissociation times of molecular unbound states are probably in the fs range [13], while the lifetimes of the possible atomic emitters are typically few ns $[14,15]$. Molecular dissociation can therefore be considered so fast that it will not affect the temporal distribution of electron-photon coincidences in our measurements. We observe the highest coincidence rate in NO around 41-eV photon energy. The possible mechanisms which can lead to the observed electron-VUV photon coincidences are decay of superexcited states and dissociative photoionization with excitation. Superexcited states have very recently been observed in the inner-valence region of the No molecule [16], but only DIE can adequately explain our observations.

\section{Experimental}

The experiments were carried out at the Gas Phase Photoemission beamline [17] at the Elettra synchrotron radiation laboratory in Trieste (Italy). The beamline uses undulator radiation that is monochromatized by a spherical grating monochromator equipped with a planar pre-mirror. After the monochromator, the photon beam can be directed to any of three branch lines. Our experiment was situated at the branch that operates in the photon energy range of $14-200 \mathrm{eV}$ and that also hosts a Ti:Sapphire laser system for two-color experiments that combine both laser and synchrotron radiation excitation. During the normal multi-bunch operation of the Elettra storage ring, electron bunches are filled in the ring so that synchrotron radiation is emitted every $2 \mathrm{~ns}$, the pulses being $\sim 60$ ps wide. However, there is a so-called "dark gap" in the filling pattern where no electron bunches are present. The length of this gap is typically $50 \mathrm{~ns}$, while the whole period of the ring is $864 \mathrm{~ns}$. During the present experimental session the storage ring operated in a special filling mode where one (or few) electron bunch (-es) was (were) injected in the dark gap. This allowed us to perform time-resolved experiments where the intensity of the fluorescence emission was measured in coincidence with the storage ring clock (one signal for each period of the electron bunches).

A threshold electron analyzer is an obvious choice for detecting photoelectrons in PEFCO experiments, as it can achieve both high transmission and high-energy resolution. However, a threshold electron analyzer detects only electrons whose kinetic energy is typically less than few meV. Not knowing the cross section of DIE at threshold, we wanted to collect low-energy electrons in a slightly larger energy range. Therefore, an electron time-of-flight (e-TOF) spectrometer was chosen for electron detection. The spectrometer, which was borrowed from the Imperial College (London, UK), was constructed according to the design of Hemmers et al. [18]. It is composed of an electronic lens system and a magnetically shielded drift tube whose length is $244 \mathrm{~mm}$. The e-TOF spectrometer was positioned along the electric vector of the incident synchrotron light. To increase the collection efficiency, photoelectrons were pushed towards the grounded input aperture of the e-TOF by applying a negative voltage on a mesh situated on the opposite side of the interaction region (IR), as it is shown in Fig. 1. An electrically isolated needle was used for the gas inlet. The ambient pressure in the chamber ranged from $2.4 \times 10^{-6}$ to $1.1 \times 10^{-5}$ mbar during the measurements, but the pressure in the IR is estimated to be 10-50 times higher. For intensity reasons the needle was brought close ( $\mathrm{ca}$. $1 \mathrm{~mm}$ ) to the IR, which resulted in the perturbation of the electric field. This was partly compensated by applying a potential also to the needle. Voltages were chosen empirically so that transmission was highest for electrons of kinetic energy $<1 \mathrm{eV}$. Electrons passing through the e-TOF spectrometer were detected with a $40-\mathrm{mm}$ diameter Z-stack micro-channel plate (MCP) detector (set of three stacked MCPs) located after the drift tube (not shown in Fig. 1).

For photon detection, the experimental setup included another MCP detector with a 25-mm diameter (a chevron configuration -

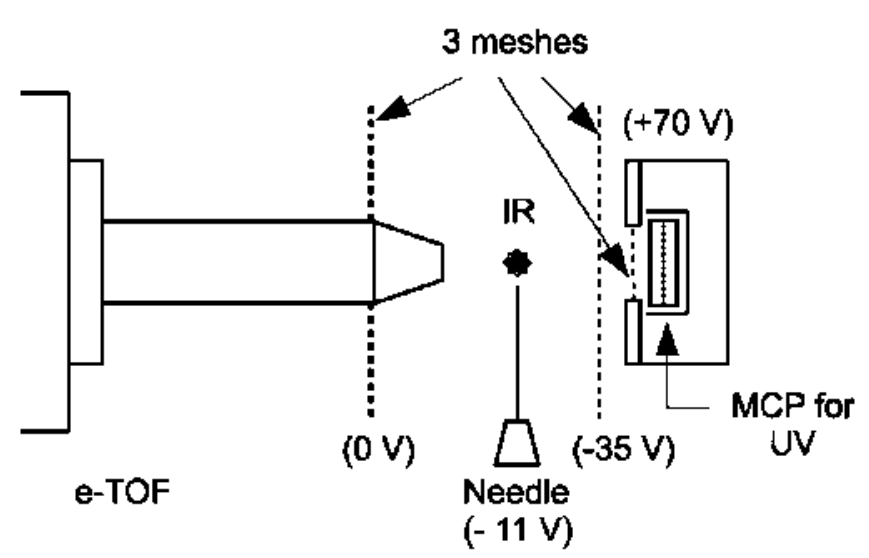

Fig. 1. Schematic presentation of the experimental artangement around the interaction region (IR). Typical potentials applied to the meshes, the needle and the cup surrounding the MCP are given in parentheses. Synchrotron light comes perpendicularly from the back side of the paper. The distance between the nose of the e-TOF spectrometer and the IR was $20 \mathrm{~mm}$. 
stack of two MCPs). It was installed opposite to the e-TOF spectrometer (Fig. 1), also in the direction of the light polarization vector. This MCP detector was shielded from the sides and back to screen it from stray photons. There was a mesh (90\% transmission) in front of the MCP. Application of a positive potential on it prevented positive ions from entering the detector. The front of the MCP was set at $-2.8 \mathrm{kV}$ (this assured that any electrons were repelled and did not contribute to the signal), while the back was at ground. The use of a standard MCP as a photon detector is limited to wavelengths below $150 \mathrm{~nm}$. Our MCP detector could measure the yield of Lyman- $\alpha$ photons $(121.6 \mathrm{~nm})$ from methane [19] but the intensity appeared weak, so the upper sensitivity limit is probably well below $150 \mathrm{~nm}$.

It was found that a part of the measured photon signal was due to scattering and reflection of the incident synchrotron light. The major cause of the reflected light was the chamber's glass window which was installed for the entrance of the laser beam in order to perform possible two-color experiments. Our efforts to reduce the reflections by masking the window with the diagnostics photo diode already mounted inside the chamber on a manipulator only worsened the situation. However, in electron-fluorescence photon coincidence measurements the scattered light contributed only to the increased level of uniform background. On the other hand, it created distinct peaks in the experiments where the arrival times of photons were recorded in coincidence with the storage ring clock.

The signals from the detectors were fed into a Time-to-Digital Converter (TDC) system (TMD-GPX from ACAM [20]) in order to record the arrival times. The TDC was configured to provide 81 -ps resolution. Even though the photon in our experiment was emitted after the correlated photoelectron, it was actually detected first because it takes some tens of ns for the electrons to pass through the e-TOF spectrometer before reaching the detector. In the electron-fluorescence photon coincidence measurements performed, the photon detector provided the start signal and the e-TOF the stop signal.

\section{Test experiments with $\mathrm{Ne}$}

Performance of the fluorescence photon-slow electron coincidence apparatus was tested with Ne atoms. The incident photon energy was scanned with a step of $\sim 25 \mathrm{meV}$ across the Ne 2 s threshold (48.475 eV [21]) and coincidences were measured between VuV photons and electrons. These coincidences, shown in the spectra of Fig. 2, come either from direct $\mathrm{Ne} 2 \mathrm{~s}$ photoionization, which is followed by $2 \mathrm{~s}^{-1} \rightarrow 2 \mathrm{p}^{-1}$ radiative decay with fluorescence emission at $\sim 46 \mathrm{~nm}[21]$, or from $\mathrm{Ne} 2 s \rightarrow$ np Rydberg excitations that converge to the 2s IP [22]. In the latter case, the excited Rydberg states were found to decay by fluorescence $(\sim 27 \mathrm{eV}$ photons $)$ to the $2 s^{2} 2 p^{5}\left({ }^{2} P_{1 / 2}\right)$ n'p states which further decayed by autoionization to the $\mathrm{Ne}^{+} 2 s^{2} 2 \mathrm{p}^{5}\left({ }^{2} \mathrm{P}_{3 / 2}\right)$ state, releasing an electron with kinetic energy of less than $97 \mathrm{meV}$. Exciting photon energies were caljbrated by comparing our VUV yield to that published in Ref. [22]. However, there remains an uncertainty of $15 \mathrm{meV}$ (or about half of the used step size) in the photon energy scale.

We can clearly see that an electron-VUV photon coincidence peak arises at the time of $\sim 32 \mathrm{~ns}$, when the exciting photon energy crosses the Ne 2s threshold. Below the 2s IP, we observe only hints of weak intensity, particularly in the second and third lowermost spectra in Fig. 2. In the former, the incident photon beam induced the $\mathrm{Ne} 2 s \rightarrow 15 \mathrm{p}$ and $16 \mathrm{p}$ transitions, while still higher Rydberg orbitals ( $n \approx 18$ ) were excited in the latter. A prominent intensity increase occurs when the photon energy exceeds the Ne $2 \mathrm{~s}$ IP. The coincidence peak is narrowest just above the threshold. It then splits in two components whose time difference increases with the excess energy above the $\mathrm{Ne} 2 \mathrm{~s}$ IP. The two peaks originate from the

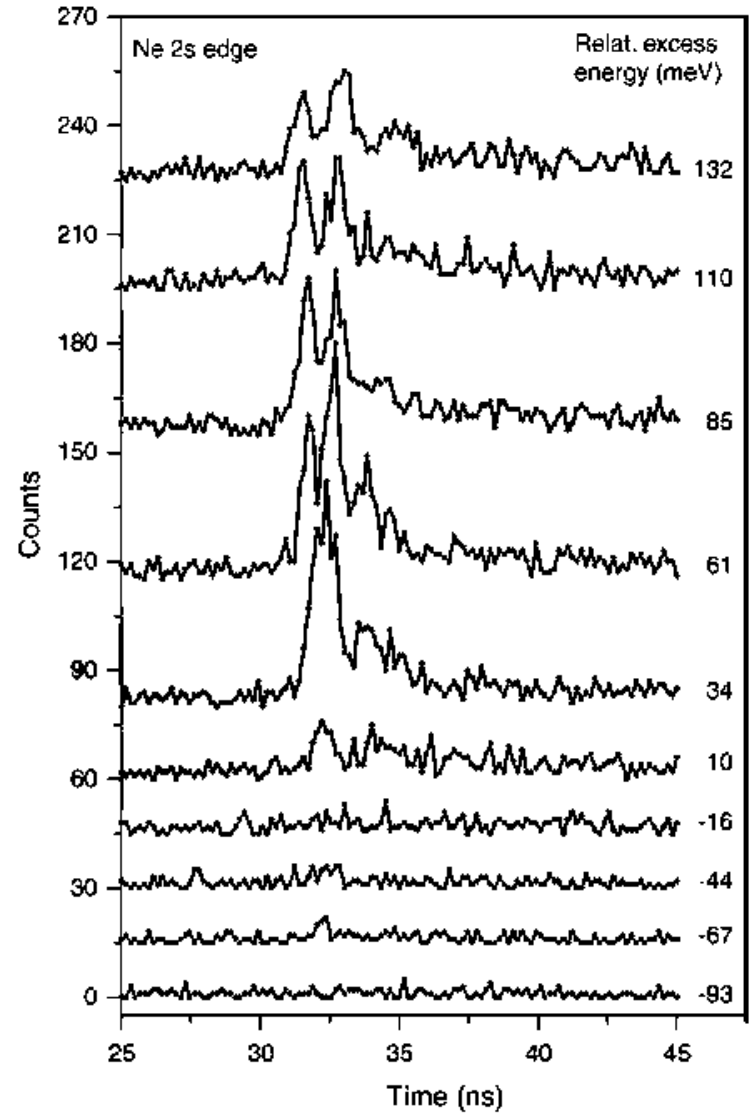

Fig. 2. Electron-VUV photon coincidence measurements at the Ne 25 ionization threshold. Acquisition time was 200 s per 5 pectrum. The base levels of the spectra lave been shitted for clarity: the real base limes are $\wedge 1$ count in eacli spectrum. Plioton energy resolution of the incident radiation was about 15 meV. Photon energies are given on the right side of each spectrum in the units of meV relative to the Ne $2 s$ IP $(48.475 \mathrm{eV})$. The uncertainty in the energies is $\pm 15 \mathrm{meV}$.

photoelectrons that are emitted towards and away from the e-TOF spectrometer. The angular anisotropy parameter, $\beta$, is 2 for $\mathrm{Ne} 2 \mathrm{~s}$ photoionization, which means that the photoelectrons are emitted preferentially along the electric vector of the linearly polarized light. The e-TOF spectrometer was positioned in that direction.

We do not observe any electron-VUV photon coincidences at the photon energies below $48.38 \mathrm{eV}$ (the lowermost spectrum in Fig. 2). This also implies that Ne 2 s photoionization caused by the second-order and higher order radiation, which is known to be present to some extent in the photon beam after the monochromator, does not disturb this particular experiment. The reason is the reduced TOF collection efficiency for fast electrons. Electrons emitted directly towards the entrance aperture of the e-TOF spectrometer should be detected no matter what is their kinetic energy but the TOF acceptance solid angle is very small $(0.06 \%$ of $4 \pi)$ for the used geometry (TOF input aperture has a $2-\mathrm{mm}$ diameter and it is $20 \mathrm{~mm}$ apart from the IR). (For low-energy electrons the collection efficiency is largely increased by application of the external electric field, reaching $100 \%$ for zero kinetic energy electrons.) Indeed, no structure is observed at time of flights shorter than $32 \mathrm{~ns}$, where high-energy photoelectron-VUV photon coincidences originating from second or higher order radiation are expected. In contrast. there is a weak structure at a time slightly longer than that of the main peak (for instance, at $\sim 34 \mathrm{~ns}$ with $61 \mathrm{meV}$ excess energy). It seems to be connected to electron-VUV photon coincidences. This "side" peak is most likely due to the ringing signal in the electron detector or amplifier. 


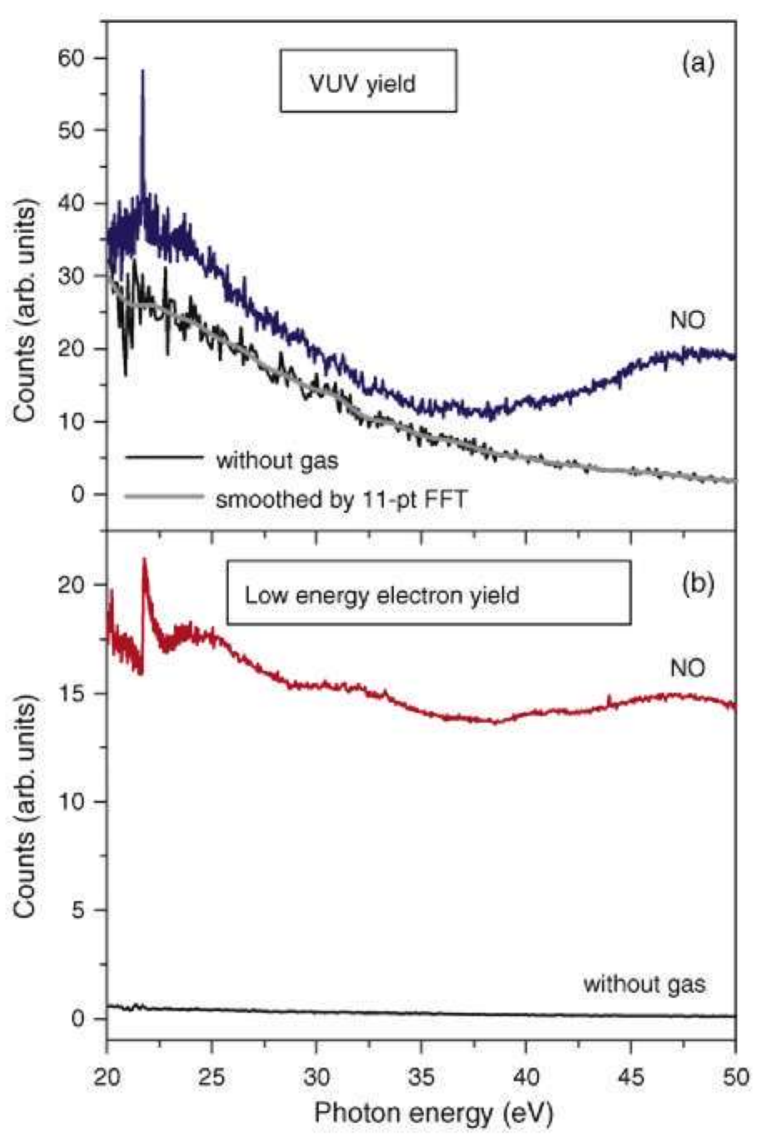

Fig. 3. (a) The total VuV fluorescence yield and (b) the total low-energy electron yield of NO as a function of exciting photon energy. The sharp peak at $21.68 \mathrm{eV}$ in (a) is due to metastable fragments [23]. The lower curves in each panel correspond to the same measurements without gas. The results have been normalized to the photodiode current.

\section{Experiments with NO}

The "total" fluorescence (limited to VUV photons) and photoelectron (low energy - see collection efficiency discussion above) yields of the NO molecule were first measured in single spectra, by registering the signals coming from the detectors without any restriction on their arrival times. The reference spectra were measured without the sample gas. These results are shown in Fig. 3. It is clear that, in the fluorescence yield measurement, most of the intensity at photon energies below $35 \mathrm{eV}$ is due to scattered light because the reference spectrum is very similar to the spectrum measured with the NO gas. However, the fluorescence yield from NO shows a sharp peak at $21.68 \mathrm{eV}$. This peak is actually not due to fluorescence, but due to metastable fragments that have been observed just before the $\mathrm{c}^{3} \Pi$ threshold of $\mathrm{NO}^{+}[23]$. Metastable fragments, drifting in all directions, can have enough internal energy to be detected when they hit the MCP. At photon energies above $35 \mathrm{eV}$, the fluorescence yield measured with the sample gas begins to have more intensity than the reference spectrum without gas, indicating that VUV emission of the NO molecule increases significantly at these excitation energies. Dispersed VUV emission in the NO molecule has been observed, for instance, at wavelengths $118-142 \mathrm{~nm}$ following the photoexcitation of the $\mathrm{N} 1 \mathrm{~s}$ and $O 1$ s electrons to the antibonding $2 \pi$ orbital [24] and at wavelengths $110-180 \mathrm{~nm}$ using electron impact at 100-eV energy [25], but, to our knowledge, not after direct inner-valence photoionization. The two spectra $[24,25]$ display quite similar intensity ratios between the vibrational bands in the $\mathrm{NO}^{+} \mathrm{A}^{1} \Pi \rightarrow \mathrm{X}^{1} \Sigma^{+}$emission system and the emission lines due to excited neutral nitrogen and oxygen fragments.

In contrast to the reference fluorescence yield, the electron signal is almost negligible in the reference measurement without gas (Fig. 3b). Most of the electrons in the IR therefore originate from the sample gas. The measured electron yield rises rapidly at $21.7 \mathrm{eV}$, at an energy that is immediately after the resonance peak due to the metastable fragments in Fig. 3a. This rise can be attributed to the opening of a new photoionization channel that leads to the $c^{3} \Pi$ state of the $\mathrm{NO}^{+}$ion. In other words, when photon energy barely exceeds the $c^{3} \Pi$ ionization potential $(21.7 \mathrm{eV}[26])$, very slow electrons are emitted and efficiently collected by the e-TOF spectrometer. The threshold photoelectron spectrum (PES) of NO [27] does not show any features immediately after the threshold of $\mathrm{c}^{3} \Pi$ photoionization. Therefore, the shape of this peak in our electron yield spectrum is concluded to represent approximately the transmission of the e-TOF spectrometer in the present measuring conditions. The FWHM of the peak is about $0.42 \mathrm{eV}$ and the total width at the base level is about $1.1 \mathrm{eV}$. Thus, the collected electrons mostly have kinetic energies less than $1 \mathrm{eV}$ in our experiments. Because of this modest electron resolution, all the sharp features that were observed in the threshold PES of NO [27] are smeared out in our spectrum. The electron yield displays a high background, which is due to slow electrons that can be created when photoelectrons lose energy when colliding with molecules, walls or other surfaces in the chamber. These secondary electrons, however, are not correlated with VUV emission and therefore do not create peaks in the coincidence spectrum. The high background (like in Fig. 3b) can be tolerated in the electron yield, as the enhanced transmission achieved should help to decrease considerably the measuring time in coincidence measurements.

Coincidences between VUV photons and slow electrons were measured at various photon energies in the valence region; the spectra are shown in Fig. 4. The coincidence peak forms at $29 \mathrm{~ns}$ when photon energy is increased. The slightly different position of the maximum as compared to the Ne measurements shown in Fig. 2 was caused by a small adjustment of the operating voltages of the e-TOF spectrometer. The measurements at 25 and $30-\mathrm{eV}$ photon energy show no peaks above noise level. In contrast, at 35-eV photon energy a very narrow peak with weak intensity appears at the correct position for electron-VUV photon coincidences. At 38-eV photon energy, the coincidence peak becomes more intense and broader. This indicates that a threshold, which leads to both electron and VUV photon emissions, has been exceeded and the electrons detected can also have a small amount of kinetic energy. The coincidence rate increases substantially when going from 38 to $41-\mathrm{eV}$ photon energy. At the latter energy, we observed about 4 coincidences/minute within a 1 -ns wide window at the peak maximum, while the background level was $\sim 0.3$ counts $/ \mathrm{min}$. (These spectra have been binned from the original data.) At $50-\mathrm{eV}$ photon energy, the coincidence rate decreases at the maximum and the peak profile becomes broader. This is obviously caused by the increase in the average kinetic energy of the photoelectrons detected.

The temporal profile of VUV emission was measured at the photon energy of $41.5 \mathrm{eV}$. The time measurement was started by a photon hitting the MCP and stopped by the ring clock, which leads to a reversed time axis in the spectrum. The temporal distribution of detected photons with and without NO gas are shown in Fig. 5 for the time region where the extra electron bunches were inserted in the dark gap (the position of the origin of the time axis, $0 \mathrm{~ns}$, is irrelevant). The inset shows the spectrum without gas in a wider time region. It demonstrates that there were no electron bunches between 210 and 180 ns until the three electron bunches inserted in the dark gap arrived (at 181.3,179.3 and $173.3 \mathrm{~ns}$ ). These measurements did not involve electron detection, hence the result obtained 


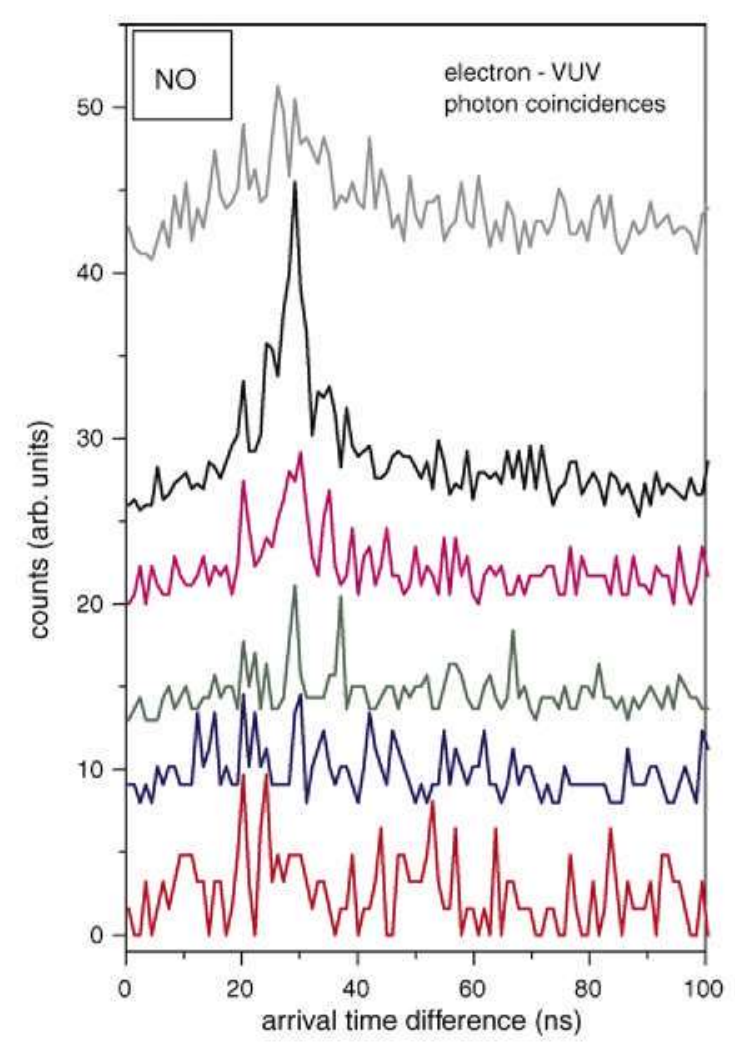

Fig. 4. The photoelectron-VUV photon coincidence spectra of NO measured at the photon energies of $50,41,38,35,30$, and $25 \mathrm{eV}$ (traces from top to bottom). The spectra have been normalized to the photon flux measured with a photodiode. Measuring time was $10 \mathrm{~min}$ for each spectrum. The base levels of spectra have been raised for clarity as follows: at $50 \mathrm{eV}$ by 40 counts, at $41 \mathrm{eV}$ by 25 counts, at $38 \mathrm{eV}$ by 20 counts, at $35 \mathrm{eV}$ by 13 counts and at $30 \mathrm{eV}$ by 9 counts.

does not necessarily relate to the coincidence events observed in Fig. 4, but to the most intense VUV emission detected by the MCP. The measurement without gas shows that there is a significant contribution of scattered SR light (probably from the needle). This can be deduced from the appearance of the peaks which fit the electron

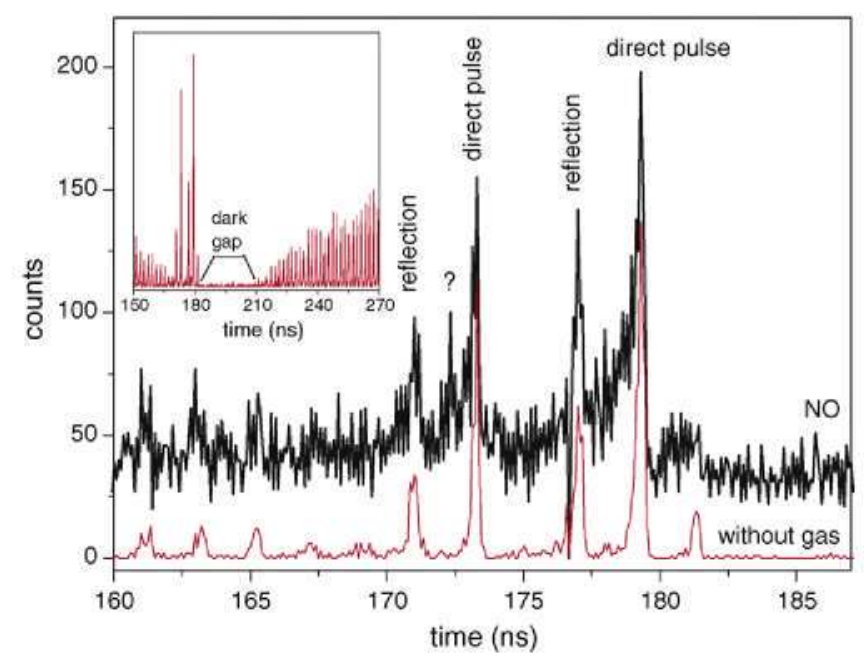

Fig. 5. VUV photon-ring clock coincidence spectra measured with and without NO gas. The inset shows the spectrum without gas in a wider region, demonstrating the electron bunch structure and the dark gap that begins at $\sim 210$ ns and ends at $\sim 170 \mathrm{~ns}$. Note that the real time axis runs from right to left. Measurement time was $60 \mathrm{~min}$ for each spectrum. Photon energy was $41.5 \mathrm{eV}$.

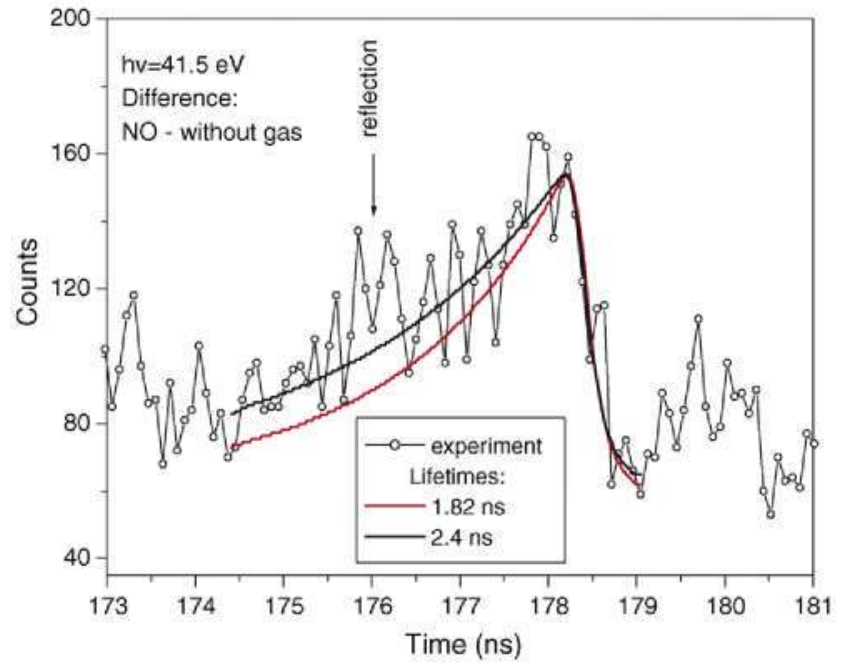

Fig. 6. The decay time analysis of VUV fluorescence emission in the NO molecule. The "instrumental" profile (see text for details) was convoluted by the exponential decay curve, characterized by the lifetimes of $1.82 \mathrm{~ns}$ (red curve) and $2.4 \mathrm{~ns}$ (black curve), and adjusted on the experimental spectrum (points connected with line), which is the difference of the two curves in Fig. 5. (For interpretation of the references to color in this figure legend, the reader is referred to the web version of the article.)

bunch time structure of the Elettra storage ring (pulse every $2.0 \mathrm{~ns}$ ). However, there were also some peaks whose intervals (2.3 ns in Fig. 5) did not follow the 2.0-ns bunch structure of the ring. As those peaks shifted upon the insertion of the photodiode in front of the glass exit window, they should be caused by the reflection of the incident SR beam when it hits the end of the beam line, i.e., the photodiode or the exit window. They are labeled as "reflection" in Fig. 5. The origin of the peak, which is provided with a question mark, is not clear.

The most intense peak at $\sim 179$ ns in the measurement with the sample gas (Fig. 5) shows an exponential tail on the shorter times, as would be expected for radiative decay that is characterized by a lifetime of the order of a couple of ns. The tail is on the left side of the peak because VUV photons start the time measurement. The time structure of the peak at 179 ns was analyzed after the subtraction of the two spectra of Fig. 5. The subtracted spectrum is shown in Fig. 6. The temporal profile in the measurement without gas is given by the incident SR pulse, whose width is about $60 \mathrm{ps}$, and by any possible broadening effects due to the detector, electronics, etc. Let us call it as an instrumental profile. We assume that the same instrumental profile is maintained in the measurement with the NO gas, in which case the peak shape is further modified by the decay of the emitting states. Accordingly, the observed line shape in the measurement with NO was taken to be the convolution of the peak observed around $179 \mathrm{~ns}$ in the measurement without gas (Fig. 5) and an exponential decay function that described the lifetime dependence of the emitting state. The largest contribution to VUV emission could come from the $\mathrm{O}$ and $\mathrm{N}$ emission lines at 130.4 and $120.0 \mathrm{~nm}$, respectively, as they are the most intense ones in the published fluorescence spectra $[24,25]$. The lifetimes of the emitting states have been determined to be $1.82( \pm 0.05) \mathrm{ns}$ for the O I line at $130.4 \mathrm{~nm} \mathrm{[14]} \mathrm{and} \sim 2.4 \mathrm{~ns}$ for the N I lines at $120.0 \mathrm{~nm}$ [15], the latter value being the average of several experiments. The results of convolution with these lifetimes are shown in Fig. 6 . The convoluted curve follows the experimental data points better when the lifetime of the emitting state is $2.4 \mathrm{~ns}$. This could imply a significant contribution from the $\mathrm{N}$ emission line at $120.0 \mathrm{~nm}$, which is due to the $2 \mathrm{p}^{2}\left({ }^{3} \mathrm{P}\right) 3 \mathrm{~s}\left({ }^{4} \mathrm{P}\right) \rightarrow 2 \mathrm{p}^{3}\left({ }^{4} \mathrm{~S}\right)$ transitions. However, this result is not conclusive, as an equally good correspondence could 
be achieved, for instance, by a sum of two decay curves, one with a slightly shorter and the other with a slightly longer lifetime. The variation in the detection efficiency of the MCP may also have an influence: perhaps photons at $130.4 \mathrm{~nm}(9.51 \mathrm{eV})$, corresponding to possibly intense emission from the excited $\mathrm{O}$ atom, are detected with much less efficiency than those from $\mathrm{N}$ at $120.0 \mathrm{~nm}(10.33 \mathrm{eV})$. There are several other atomic emission lines in the VUV range of interest; the calculated lifetimes of their upper states were tabulated in [16]. Note that the emission system $A^{1} \Pi \rightarrow X^{1} \Sigma^{+}$of the $\mathrm{NO}^{+}$ion as the origin of the decay profile is excluded because the lifetimes of the vibrationally "resolved" upper states are about 50 ns or longer $[25,28]$. Nevertheless, this molecular emission probably creates considerable background in the spectra of Figs. 5 and 6.

\section{Discussion}

We first exclude the possibility that the observed electron-VUV photon coincidences originate from photoionization of the $\mathrm{NO}^{+} \mathrm{A}$ ${ }^{1} \Pi$ state, followed by the $A^{1} \Pi \rightarrow X^{1} \Sigma^{+}$emission. The ionization energy of the $A^{1} \Pi$ state is $18.3 \mathrm{eV}$ [26]. Therefore the photoelectrons created have relatively high kinetic energies $(\sim 20 \mathrm{eV})$ in the excitation energy of interest and they are not collected efficiently. In fact, we do not see any coincidences at 25 and $30 \mathrm{eV}$ photon energies (Fig. 4), where the $\mathrm{NO}^{+} \mathrm{A}^{1} \Pi$ photoionization channel is open. No other molecular emission in $\mathrm{NO}^{+}$was observed in the wavelength region below $150 \mathrm{~nm}[24,25]$.

The VUV photons observed in coincidence with slow electrons could, in principle, originate either from decay of doubly excited (superexcited) states or from dissociative photoionization with excitation. We consider first double excitations, taking into account only those decay channels that lead to VUV emission. A doubly excited state could autoionize to a state of $\mathrm{NO}^{+}$which further fluoresces in the VUV region. The $\mathrm{A}^{1} \Pi-\mathrm{X}^{1} \Sigma^{+}$transition in $\mathrm{NO}^{+}$ emits VUV light that can be detected by our MCP. Some doubly excited states could obviously decay to the $A^{1} \Pi$ state. If such a doubly excited state were, for instance, at 38-eV excitation energy. the electron emitted would have a kinetic energy of about $20 \mathrm{eV}$. However, such a fast electron would not create a sharp peak in the electron-VUV photon coincidence spectrum. Possible decay channels involving other molecular emission of $\mathrm{NO}^{+}$in the VUV region should be much weaker, if they exist at all, and can also be neglected for the same reason that electrons emitted would have too high kinetic energies.

Other possible VUV emitters are produced when a doubly excited state dissociates into two excited neutral fragments. If both fragments fluoresce, as it was actually observed for NO by Odagiri et al. [16], no electron is coming out. Therefore this channel cannot explain the electron-VUV photon coincidences. However, if one of the fragments autoionizes, it provides an electron for coincidence events. Hikosaka et al. [29] have observed autoionization in the $\mathrm{N}$ atom after excitation of NO to superexcited states at photon energies between 21.5 and $27 \mathrm{eV}$. Autoionization peaks lie in the electron spectrum at low kinetic energies between 0 and $2 \mathrm{eV}$, so such electrons would be efficiently collected by the e-TOF spectrometer. Superexcited states of NO have also been observed at $\sim 33-\mathrm{eV}$ excitation energy [30,31] and recently at even higher energies up to $47 \mathrm{eV}[16]$. However, we can give some arguments suggesting that this channel is not responsible for the detected electron-VUV photon coincidences. Superexcited states are of resonant nature and do not give rise to a smooth intensity distribution at all excitation energies above a given threshold, as it is observed in the VUV yield of Fig. 3. The existence of "suitable" superexcited states across the whole inner-valence region is highly unlikely. Additionally, following superexcitation of NO, autoionization was observed only in the $\mathrm{N}$ fragments [29], which implies that VUV emission should take place in the $O$ atom. Our decay time analy- sis rather points out that most VUV emission comes from $\mathrm{N}$ atoms. Finally, autoionization of a fragment is typically characterized by constant electron kinetic energies as a function of the excitation energy. In contrast, the coincidence peak shape in our measurements varies with the photon energy (Fig. 4), which suggests that an electron is produced by photoionization rather than by autoionization. We therefore conclude that superexcited states are extremely unlikely to be the origin of the observed coincidences between slow electrons and VUV photons.

Remembering the transmission behavior of the e-TOF spectrometer under the present measuring conditions, the electrons detected in the electron-VUV photon coincidence spectrum at 41 $\mathrm{eV}$ photon energy (the one with highest intensity in Fig. 4) should originate predominantly from photoionization channels whose ionization energies are between 40 and $41 \mathrm{eV}$. The photoelectron spectrum of NO [26,32] shows a wide peak whose maximum is at the binding energy of $40.4 \mathrm{eV}$. It was originally assigned to primary photoionization from the $3 \sigma$ orbital [32], but the calculations of Honjou et al. [33] attributed the intensity to the states having mostly $2 \mathrm{~h}-1 \mathrm{e}$ character and to a lesser extent $3 \sigma^{-1}$ character, i.e., to the correlation satellite states. These inner-valence photoionization processes are also visible as a very weak structure between 39 and $42 \mathrm{eV}$ in Fig. 3b and partly also in the threshold PES of Ref. [27], which was measured only up to $40 \mathrm{eV}$. The latter spectrum proves that there is a continuous supply of slow electrons available also for our coincidence measurements at 35 and 38-eV photon energy. The last satellite state in the PES appears at $\sim 45-\mathrm{eV}$ ionization energy [26]. At the photon energy of $50 \mathrm{eV}$ (Fig. 4), very few electrons may therefore have less than 5-eV kinetic energy, which would explain the spreading of the coincidence peak. According to the calculations presented in [27], inner-valence states are characterized by dissociative potential energy curves that correlate with excited neutral and jonic fragments. Fluorescence emission can therefore be expected to follow inner-valence photoionization in NO. The basic features of our electron-VUV photon coincidence spectra can be accounted for if DIE takes place in the context of photoionization to $2 \mathrm{~h}-1 \mathrm{p}$ states. This interpretation agrees with the one made for inner-valence jonization of the $\mathrm{N}_{2}$ molecule [6,7]. The analogous effect has also been suggested to occur in NO with electron impact excitation [25]. The N I emission at $120.0 \mathrm{~nm}$ resulting from a DIE process is energetically possible at excitation energies above $30.45 \mathrm{eV}[21,34]$. This energy limit is well below the photon energies where we observe the electron-VUV photon coincidences.

There is a variation of DIE that produces an excited ion and a neutral atom in the ground or lowly excited state. Ehresmann et al. [35] observed N II emission lines at 91.6 and $108 \mathrm{~nm}$ in the $\mathrm{N}_{2}$ molecule using synchrotron excitation between 37 and $69 \mathrm{eV}$ photon energies. They attributed these lines to DIE. The transitions around $108 \mathrm{~nm}$ could also contribute to VUV emission observed in the present study, as the lifetime of their upper states is about $2.7 \mathrm{~ns}$ [36]. The dissociation limit in the NO molecule to produce the fragment in question, $\mathrm{N}^{+}\left(2 \mathrm{~s} 2 \mathrm{p}^{3},{ }^{3} \mathrm{D}\right)$, together with the ground-state $\mathrm{O}$ atom lies at $32.47 \mathrm{eV}[21,34]$. Similar DJE processes involving emission in $\mathrm{O}^{+}$ions appear less likely, as transitions having a wavelength below $150 \mathrm{~nm}$ and the excitation energy of the upper state less than $20 \mathrm{eV}$ are spin-forbidden except for the $O \mathrm{ll}$ lines around $83 \mathrm{~nm}$ [21]. The upper states of these transitions have $\sim 14.9 \mathrm{eV}$ excitation energy [21] (which sets the corresponding dissociation limit at about 35.0 eV [34]) and $\sim 1.2$ ns lifetime [37].

Finally, we consider expected similarities in fluorescence emission of NO following the ( $\mathrm{N}$ and $\mathrm{O}$ ) $1 \mathrm{~s} \rightarrow 2 \pi$ excitations, studied in [24], and after inner-valence photoionization. The core-excited states decay predominantly via spectator transitions to such $2 \mathrm{~b}-1 \mathrm{e}$ final states where the $2 \pi$ orbital remains occupied. These final states of spectator decay are similar to the correlation satellites in the inner-valence photoionization. In fact, according to the- 
ory [33], the most important $2 \mathrm{~h}-1$ e configurations that mix with the inner-valence state $3 \sigma^{-1}$ have the $2 \pi$ orbital occupied. We expect therefore that dissociation of the $2 \mathrm{~h}-1 \mathrm{e}$ states yields the same excited fragments after spectator decay of the (N/O $1 \mathrm{~s})^{-1} 2 \pi^{1}$ states and after direct inner-valence photoionization. The decay time analysis performed in Fig. 6 gives partial support to this idea, as it suggests a significant contribution from the $\mathrm{N} l$ emission line at $120.0 \mathrm{~nm}$. The same emission line was observed to be the most intense one after core excitation of NO [24].

\section{Conclusions}

We have performed exploratory electron-VUV photon coincidence experiments using an electron TOF spectrometer for electron detection and an MCP detector for VUV photon detection. The performance of the setup was successfully tested at the Ne 2s edge, where the emission of the $2 \mathrm{~s}$ photoelectron is followed by VUV emission due to the $2 \mathrm{~s}^{-1} \rightarrow 2 \mathrm{p}^{-1}$ transitions. Slow electron-Vuv photon coincidences were also detected in the inner-valence region of the NO molecule. The highest intensity was related to the photoelectron band around the ionization energy of $40 \mathrm{eV}$. This band has been previously associated with the correlation satellite states (2h-1e states) that mix with the inner-valence single-hole state $3 \sigma^{-1}$ of NO. These highly excited states of $\mathrm{NO}^{+}$dissociate mostly into $\mathrm{O}^{+}+\mathrm{N}^{*}$ and $\mathrm{N}^{+}+\mathrm{O}^{*}$. Excited neutral $\mathrm{O}$ and $\mathrm{N}$ atoms have transitions in the VUV range that is detected in the present experiment $(\lambda<150 \mathrm{~nm})$. The temporal behavior of VUV emission was also measured. An important part of VUV emission could be explained with a decay time of $\sim 2.4 \mathrm{~ns}$, which together with intensity considerations points out to the neutral excited $\mathrm{N}$ atom emitting at $120.0 \mathrm{~nm}$ as the most important emitter.

\section{Acknowledgments}

The authors are very grateful to L.J. Frasinski (Imperial College, London) for the possibility to use his electron TOF spectrometer. E. Karanzoulis and the whole machine group of Sincrotrone Trieste are thanked for providing us with the special filling mode of the Elettra storage ring. AK, MC and MdS acknowledge the Italian National Research Council (CNR) for funding their research activity at the Gas Phase beamline, Elettra, under projects MD.P06.016.002 (CNR-IOM) and MD.P03.026.001 (CNR-IMIP). LP acknowledges funding from the Research Council of University of Oulu. Finland. The research leading to these results has also received funding from the European Community's Seventh Framework Programme (FP7/2007-2013) under grant agreement no. 226716.

\section{References}

I1 Y. Hatano, Phys. Rep. 313 (1999) 109

|2| M. Ukai, K. Kameta, N. Kouchi, Y. Hatano. Pliys. Rev. A 46 (1992) 7019.

13] E. Meleto Garcia, J. Alvarez Ruiz, P. Erman, A. Kivimăki, E. Raclılew-Källıe, J Rius i Riu, M. Stankiewicz, L. Veseth, Chem. Phys. 293 (2003) 65.

|4| M. Kato. T, Odagiti, K. Kodama, M. Mucata, K. Kameta, N. Kouclii, J. Phys, B 37 (2004) 3127 .

I5I C.Y.R. Wu, T.S. Chien, C.C. Kim, D.L. Judge, Chem. Phys. Lett. 145 (1988) 418.

|6| M. Kitajima, M. Ukai, S. Machida, K. Kameta, N. Kouchi, Y. Hatano, T. Hayaishi, K. Ito, J. Phys. B 28 ( 1995 ) L185.

17| M. Kitajima, M. Ukai, S. Machida, K. Kameta, A, Ehresmann, N. Kouchi, Y, Hatano, T. Hayaishi, E. Shigemasa, K. Ito, J. Phys. B 29 (1996) 1711.

|8| M. Bloch, D.W. Turner, Chem. Phys. Lett. 30 (1975) 344.

|9| H. Bielil, K.।. Boyle, D.M. Smith, R.P. Tuckett, Chem. Phys, 214 (1997) 357.

|10| S. Taylor, J.H.D. Eland, Chem. Phys, 315 (2005) \&.

111 S. Taylot, J.H.D. Eland, M. Hochlaf, J. Chem. Phys. 124 (2006) 204319.

|12| S. Taylor, J.H.D. Eland, M. Hochlaf, Chem. Phys. 330 (2006) 16.

113| A. Stolow, A.E. Bragg, D.M. Neumark, Chem. Rev. 104 (2004) 1719

|14| G.M. Lawrence, Phys. Rev. A 2 (1970) 397.

I15| M. Tong, C. Froese Fischer, L. Sturesson, J. Phys. B 27 (1994) 4819.

16| T. Odagiri, K Funatsu, T. Tanabe, I.H. Suzuki, M. Kitajima, N. Kouchi, J. Phys, B $42(2009) 225101$

|17| K.C. Prince, et al., J. Synchrotron Radiat. 5 (1998) 565.

|18| O. Hemmers, S.B. Whitfield, P. Glans, H. Wang. D.W. Lindle, R. Wehlitz, I.A. Sellin, Rev. Sci. Int rum. 69 (1998) 3809 .

|19| H. Fukuzawa, T. Odagiri, T, Nakazato, M. Murata, H, Miyagi, N. Kouclii, J. Phys. B $38(2005) 565$.

120| ACAM Messelectronic GmBH. www.acam.de.

121] Yu. Ralchenko, A.E. Kramida, J. Reader, J. NIST ASD Team. NIST Atomic Spectra Database, 2008. Available from: http://physics. nist.gov/asd3.

|22| P. Lablanquie, et al., Pliys. Rev. Lett. 84 (2000) 431.

|23| Y. Hikosaka, P. Lablanquie, M. Alımad, F. Penent, J.H.D. Eland, R.I, Hall, J. Phys. B $37(2004) 283$.

|24| A, Eliresmann, W. Kielich, L Werner, Plı, V. Demekhin, D. V. Omel'yanenko, V.L. Sukhonıkov, K.-H. Scharner, H. Schmoranzer, Eur. Phys. J. D 45 (2007) 235.

[25] J.E. Mentall, H,D. Morgan, J. Cliem. Phys. 56 (1972) 2271.

|26| H.-J. Freund, H. Kossmann, V. Schmidt, Cliem. Pliys. Lett, 137 (1987) 425

|27| Y. Hikosaka, T. Aoto, K. Ito, Y. Terasaka, R. Hìrayama, E. Miyoshì, J. Chem. Phys. $128(2008) 044320$.

[28] J.E. Hesser, J. Chem. Phys, $48(1968) 2518$.

|29| Y. Hikosaka, H. Hattori, T. Hikida, K Mitsuke, J. Chem. Phys. 105 (1996) 6367.

[30| J.S. Lee, J. Chem. Pliys, 67 (1977) 3998.

1311 J.-M. Sun, Z.-P. Zhong, L.-F. Zhu, W.-B. Li, X.-J. Liu, Z.-S. Yuan, K.-Z. Xu, Phys. Rev. A 70 (2004) 012708 .

|32| K. Siegbahn, et al., ESCA Applied to Free Molecules, North-Holland, Amsterdam, 1969.

133| N. Honjou, T. Sasajima, F. Sasaki, Chem. Pliys, 57 (1981) 475.

|34| J.H.D. Eland, E.J. Duerr, Chem. Phys. 229 (1998) 1.

135| A. Eluresmann, S. Macliida, M. Kitajima, M. Ukai, K. Kameta, N. Kouchi, Y. Hatano, E. Shigemasa, T. Hayaishi, J. Phys. B $33(2000) 473$.

|36| K,L, Bell, A. Hibbert, R,P. Statfotd, Pliys. Sct, 52 (1995) 240.

|37| K,L. Bell, A. Hibbert, R.P. Statford, B.M. McLaughlin, Pliys, Scr, 50 (1994) 343. 